\title{
Gestión del talento humano en micro, pequeñas y medianas empresas de servicios en Colombia: aproximaciones a un modelo de diagnóstico organizacional ${ }^{1}$
}

DOI: https://doi.org/10.21158/01208160.n87.2019.2441

Gabriela Pantoja-Kauffmann ${ }^{2}$ Universidad Tecnológica Metropolitana gpantojakauffmann@gmail.com

Fecha de recepción: 04 de junio de 2019

Fecha de aprobación: 17 de diciembre de 2019

Cómo citar este artículo / To reference this article / Comment citer cet article / Para citar este artigo:

Pantoja-Kauffmann, G. (2019). Gestión del talento humano en micro, pequeñas y medianas empresas de servicios en Colombia: aproximaciones a un modelo de diagnóstico organizacional. Revista Escuela de Administración de Negocios, (87), 13-26. DOI: https:// doi.org/10.21158/01208160.n87.2019.2441

\section{Resumen}

El presente artículo pretende establecer una reflexión sobre el estado del arte de la gestión del talento humano para micro, pequeñas y medianas empresas - mipymes - en Colombia, en el contexto de la elaboración de un modelo de diagnóstico organizacional del sector servicios y a partir de la investigación documental sobre las prácticas de gestión del talento humano del grupo de investigación de la Facultad de Ciencias Económicas y Administrativas de la Universidad de San Buenaventura con sede en Bogotá. En este escrito se busca establecer una aproximación a un modelo de diagnóstico organizacional con el fin de determinar la contribución de las áreas de gestión del talento humano en mipymes, a partir de los componentes de un modelo de gestión humana que mide la propuesta de valor de las áreas de gestión del talento en empresas colombianas; entendiendo que la gestión del talento humano en las organizaciones que agrega valor a la operación del negocio es aquella por la cual se logra trascender los asuntos operativos y contingentes del día a día y aportar a la toma de decisiones estratégicas en función de la comprensión de los mercados empresariales. Así pues, se concluye que en las mipymes colombianas las áreas de gestión del talento humano pueden erigirse como una herramienta movilizadora de recursos internos y en fuente de ventaja competitiva, en la medida en que se integren, de forma entrelazada, en el propósito de generar valor en la organización como un sistema integral.

Palabras clave: gestión del talento humano; modelo de diagnóstico organizacional; gestión humana en mipymes; administración de recursos humanos; gestión humana en sector servicios; modelo de gestión humana.

\footnotetext{
${ }^{1}$ Artículo de reflexión desarrollado como parte del trabajo realizado como becaria del Programa de Movilidad Estudiantil y Académica de la Alianza del Pacífico, Convocatoria 2019, Programa de Administración de Empresas, Facultad Ciencias Económicas y Administrativas, Universidad de San Buenaventura, sede Bogotá (Colombia), entre febrero y abril del 2019.

${ }^{2}$ Asistente Social de la Universidad Tecnológica Metropolitana, UTEM. MBA, Magíster en Administración por la Universidad de Chile. ORCID: https://orcid.org/0000-0002-1399-4329
} 


\title{
Human talent management in micro, small, and medium service companies in Colombia: approaches to an organizational diagnosis model
}

\begin{abstract}
This article aims at establishing a reflection on the state of the art of the Human Talent Management for micro, small, and medium enterprises (MSMEs) in Colombia; in the context of the development of an organizational diagnostic model for the service sector, and on the basis of the documentary research on human talent management practices from the research group of the Faculty of Economics and Administrative Sciences in the University of San Buenaventura, Bogotá. The document attempts to establish an approach to an organizational diagnosis model with a view to determine the contribution of the areas of human talent management in MSMEs, based on the components of a human management model that measures the value proposal of the areas of talent management in Colombian companies; understanding that the human talent management in organizations adds value to the business operation when it makes it possible to transcend the operational and contingent issues of the day-to-day activity, and contributes to strategic decision making based on the comprehension of business markets. Thus, it is concluded that the areas of human talent management in Colombian MSMEs can become a tool to mobilize internal resources and in a source of competitive advantage, to the extent that they are integrated, in an interlocked way, in purpose to generate value in the organization as an integral system.
\end{abstract}

Keywords: human talent management; organizational diagnosis model; human management in MSMEs; human resources administration; human management in the service sector; human management model.

\section{Gestão de talentos humanos em micro, pequenas e médias empresas de serviços da Colômbia: abordagens para um modelo de diagnóstico organizacional}

\section{Resumo}

Este artigo tem como objetivo estabelecer uma reflexão sobre o estado de arte da gestão de talentos humanos para micro, pequenas e médias empresas (MPEs) na Colômbia, no contexto da elaboração de um modelo de diagnóstico organizacional do setor de serviços e a partir da pesquisa documental sobre as práticas de gestão de talentos humanos do grupo de pesquisa da Faculdade de Ciências Econômicas e Administrativas da Universidade de San Buenaventura, com sede em Bogotá.

Este artigo procura estabelecer uma aproximacao a um modelo de diagnóstico organizacional a fim de determinar a contribuição das áreas de gerenciamento de talentos humanos nas MPEs, com base nos componentes de um modelo de gerenciamento humano que mede a proposta de valor das áreas de gestão de talentos nas empresas colombianas; entendendo que a gestão do talento humano nas organizações que agrega valor à operação dos negócios é aquela pela qual é possível transcender as questões operacionais e contingentes do dia-a-dia e contribuir para a tomada de decisões estratégicas com base no entendimento do mercados de negócios. Assim, conclui-se que nas MPEs colombianas as áreas de gestão de talentos humanos podem se tornar uma ferramenta para mobilizar recursos internos e uma fonte de vantagem competitiva, na medida em que se integrem com o objetivo de gerar valor na organização como um sistema abrangente.

Palavras-chave: gestão de talentos humanos; modelo de diagnóstico organizacional; gestão humana em MPEs; administração de recursos humanos; gestão humana no setor de serviços; modelo de gestão humana. 


\section{Gestion des ressources humaines des petites et moyennes entreprises colombiennes du secteur des services: approches d'un modèle de diagnostic organisationnel}

\section{Résumé}

Cet article vise à établir une réflexion sur la gestion des ressources humaines pour les petites et moyennes entreprises - PME - en Colombie, dans le cadre du développement d'un modèle de diagnostic organisationnel du secteur des services, et, d'une recherche documentaire sur les pratiques de gestion des ressources humaines du groupe de recherche de la Faculté de Sciences Économiques et Administratives de l'Université de San Buenaventura de Bogotá. Cet article tente d'établir un modèle de diagnostic organisationnel afin de déterminer la contribution des domaines de gestion des ressources humaines des PME sur la base des composants d'un modèle de gestion mesurant la qualité des départements de ressources humaines des entreprises colombiennes. La gestion correcte des R.H. dans les organisations apporte de la valeur au fonctionnement de l'entreprise et permet de transcender les questions opérationnelles du quotidien en contribuant à la prise de décision stratégique basée sur la compréhension du modéle économique de l'entreprise. En conclusion, pour les PME colombiennes, la gestion des ressources humaines est un outil mobilisateur de ressources internes et devient une source d'avantage concurrentiel, dans la mesure où elles sont intégrées dans l'organisation en tant que système intégral générateur de valeur.

Mots-clés: gestion des ressources humaines; modèle de diagnostic organisationnel; gestion des ressources humaines dans les PME; gestion des ressources humaines dans le secteur des services; modèle de gestion des ressources humaines. 


\section{Introducción}

Ta administración de recursos humanos de modo tal que se obtenga el mayor rendimiento posible. Esto involucra los procesos administrativos asociados, tales como contratos, permisos administrativos, registro de asistencia, vacaciones, evaluaciones, etc. Por su parte, la gestión estratégica del talento humano o la gestión humana, implica ubicar al individuo en el centro del quehacer organizacional $y$, por ende, generar iniciativas para su desarrollo. Lo anterior no desconoce los procesos administrativos inherentes, pero supera la noción de administrar.

La gestión del talento humano en una organización está llamada, en primer lugar, a conseguir un adecuado dimensionamiento de la dotación de las personas, es decir, analizar, reflexionar y desarrollar políticas destinadas a disponer del capital humano en el sentido cuantitativo y cualitativo preciso. Así, entonces, desde un punto de vista estratégico, las áreas de gestión del talento humano pueden aportar de manera significativa a los resultados de las empresas mediante la generación y la mantención de las ventajas competitivas, aspecto indispensable para la sobrevivencia y el desarrollo en el mercado.

Ahora bien, en las mipymes se encuentran rasgos que constituyen nuevas ventajas competitivas en comparación con las grandes empresas. Una de ellas es su flexibilidad, la cual permite una adaptación rápida frente a los cambios del entorno y mayor orientación al mercado (Camisón, 1996). Del mismo modo, la flexibilidad de las mipymes posibilita una mayor proximidad al cliente y una respuesta más rápida y exacta a sus necesidades.

A pesar de esto, las mipymes presentan importantes deficiencias en su planificación y práctica estratégica, de manera que es esta una de las razones de su fracaso en el desarrollo de sus activos, principalmente los intangibles.

El presente artículo busca establecer tres reflexiones con una base teórico-práctica sobre la gestión del talento humano en las mipymes de servicios colombianos. Así, primero, reflexiona sobre la relevancia estratégica de la gestión del talento humano en las organizaciones. Segundo, establece las principales características que asume la gestión del talento humano en las mipymes colombianas y plantea algunas reflexiones sobre la contribución de la gestión del talento humano desde la teoría de los recursos y las capacidades. Finalmente, pretende entregar una aproximación a un modelo de diagnóstico organizacional que sirva como base para establecer la contribución de las áreas de gestión del talento humano, para lo cual toma como punto de partida las dimensiones de un modelo de gestión humana que mide el aporte de valor de las áreas de gestión del talento al desarrollo de las empresas.

\section{Objetivo y metodología}

\begin{abstract}
A partir de una revisión bibliográfica con relación Aa los principales estudios sobre gestión del talento humano, el objetivo de este escrito es generar una reflexión que busca establecer un estado del arte en torno a la gestión del talento humano
\end{abstract}

en mipymes en Colombia, para luego aportar con insumos al proceso de elaboración de un modelo de diagnóstico organizacional que permita medir la contribución de las áreas de gestión del talento humano a la estrategia organizacional. 


\section{Resultados}

\subsection{Relevancia de la gestión del talento humano en las organizaciones modernas}

Durante décadas, la gestión del talento humano o gestión humana, también denominada «administración de personal y recursos humanos», se vinculó a prácticas administrativas tales como la contratación, las remuneraciones, el control de asistencia, etc. Todas prácticas orientadas a realizar funciones de control y mejoramiento de técnicas de estos procesos (Calderón, Naranjo y Álvarez, 2010) a partir de la organización de las personas con el fin de optimizar al máximo el rendimiento humano en el trabajo.

Sin embargo, la complejidad del entorno condujo a las organizaciones a evolucionar y modernizar sus estructuras, sus roles y sus prácticas internas, de modo que generaran respuestas adaptativas y de valor para las compañías (Calderón et al., 2010). Estas nuevas expresiones organizacionales de carácter estratégico se han abordado profusamente desde las ciencias administrativas por autores clásicos como Hill y Jones (1996), Hitt, Ireland y Hoskisson (1999), Pfeffer (2000), o Koontz, Weihrich y Cannice (2004).

La evolución de los mercados y las transformaciones generadas en el contexto empresarial y social produjeron también importantes cambios en la concepción de la contribución de las personas a las organizaciones. Así, las áreas de recursos humanos se convirtieron en áreas de gestión humana, talento humano o gestión y desarrollo de las personas como se conoce actualmente en otros países del mundo y Latinoamérica - relevantes para el logro de las metas organizacionales y de generación de ventajas competitivas (Cuesta, 2017).

Sobre la evolución de la gestión humana en las organizaciones, Calderón, Naranjo y Álvarez (2011) plantean que la gestión en las empresas implica trascender los asuntos operativos y contingen- tes del día a día, con lo cual aluden a decisiones y acciones estratégicas y a logros de objetivos de largo plazo mediante la cadena de valor interna que genera la productividad. En relación con el rol de la gestión del talento humano en las organizaciones, Tejada añade, a lo establecido por Calderón et al. (2011), que, «desde la perspectiva organizacional, la gestión del talento se basa en la legitimización de que la potencialidad humana se puede relacionar con los procesos productivos eficientes, eficaces y efectivos» (2003, p. 117). De ahí que Barney y Wright (como se citan en Calderón, 2008) establezcan que el área de gestión humana se considera un recurso organizacional clasificado dentro de los activos intangibles e importantes en el logro de las capacidades distintivas de las empresas.

De acuerdo con Calderón (2008), las organizaciones de alto rendimiento con áreas de gestión del talento que agregan valor son aquellas que cuentan con estructuras flexibles y prácticas de gestión de personas innovadoras. Este tipo de organizaciones se orientan a la mantención y retención de los empleados, con el propósito de aumentar la productividad y reducir costos. De esta forma, cuando la gerencia y las jefaturas de la organización se preocupan por las personas, sus motivaciones y su desarrollo, crean oportunidades para la promoción justa e imparcial, y potencian así la formación de competencias de las trabajadoras y los trabajadores. Esto influye de forma directa en el mejoramiento de los resultados del negocio.

No obstante, las áreas de gestión del talento humano pueden presentar dificultades para agregar valor a las organizaciones si no cuentan con el apoyo real de la gerencia, si tienen limitaciones de autonomía o si la organización no está convencida de su importancia estratégica, o bien, son reactivas y centradas en la cotidianidad operativa y tienen dificultades para traducir en cifras la efectividad del área (Calderón, 2008; Ulrich, 1998). 
Las investigaciones en Colombia sobre la contribución de las áreas de gestión del talento humano a la estrategia organizacional son escasas. Sin embargo, de los estudios publicados se destaca, particularmente, uno realizado por Gregorio Calderón en el 2008 con 109 empresas afiliadas a la Asociación Colombiana de Gestión Humana-Acrip, gremio que integra 530 empresas de diferentes sectores y tamaños, distribuidas en todo el país, con excepción de Antioquia, y cuenta con una asociación propia, que es Ascort. Esta investigación concluyó que las áreas de gestión humana generan valor a las organizaciones en cinco dimensiones: proyección organizacional —orientación estratégica一, gestión del cambio, infraestructura organizacional eficiencia y eficacia-, liderazgo de las personas y responsabilidad social (Calderón et al., 2010).

En síntesis, la gestión del talento humano en las organizaciones que agrega valor a la operación del negocio es aquella por la cual se logra trascender los asuntos operativos y contingentes del día a día y aportar a la toma de decisiones estratégicas en función de la comprensión de los mercados empresariales.

\subsection{Caracterización de las micro, pequeñas y medianas empresas de servicios en Colombia}

La Ley 905 de 2004 sobre promoción del desarrollo de las mipymes, micro, pequeñas y medianas empresas colombianas las define, en su artículo 2, en los siguientes términos:

Toda unidad de explotación económica realizada por una persona natural o jurídica en actividades empresariales, agropecuarias, industriales, comerciales o de servicios, rurales o urbanas, que responda a dos (2) de los siguientes parámetros:

Mediana empresa: a) Planta de personal entre cincuentayuno (51)ydoscientos (200) trabajadores, o b) Activos totales por valor entre cinco mil uno (5.001) a treinta mil (30.000) salarios mínimos mensuales legales vigentes; 2 . Pequeña empresa: a) Planta de personal entre once (11) y cincuenta (50) trabajadores, o b) Activos totales por valor entre quinientos uno (501) y menos de cinco mil (5.000) con salarios mínimos mensuales legales vigentes o, 3. Microempresa: a) Planta de personal no superior a los diez (10) trabajadores o, b) Activos totales excluida la vivienda por valor inferior a quinientos (500) salarios mínimos mensuales legales vigentes.

Según el Censo Económico de 2005 del Departamento Administrativo Nacional de Estadísticas-DANE (Ministerio de Comercio, Industria y Turismo y Viceministerio de Desarrollo Empresarial, 2008), las micros, pequeñas y medianas empresas representan un $80,8 \%$ del empleo en Colombia, a diferencia de las grandes empresas que solo generan el $19,2 \%$.

Tradicionalmente, se pensaba que el ideal empresarial era la creación de grandes estructuras, complejas y jerarquizadas, sin embargo, las investigaciones de la última década en industrias japonesas e italianas han conducido a cambiar el paradigma para Latinoamérica y países desarrollados ya que los negocios jóvenes y pequeños poseen potencialidades que se deben explotar (Sánchez y Giraldo, 2008). En comparación con las empresas grandes, las pequeñas y medianas empresas aportan una mayor flexibilidad para adaptarse a los cambios tecnológicos y a las distintas fases del ciclo económico (2008). Por esto se señala que, «en el campo empresarial, se destaca la importancia y dinamismo de las micro, pequeñas y medianas empresas dentro del tejido socioeconómico de cualquier país, en variables como número de establecimientos, empleo, producción, distribución del ingreso, entre otros indicadores» (Vera y Mora, 2011, p. 214).

En los países en vías de desarrollo la mayor parte de las oportunidades de empleo y de generación de ingresos dependen de las mipymes, las cuales muchas veces se consideran el principal mitigador de la pobreza, pues su flexibilidad y su especialización también pueden contribuir a la diversificación de los sistemas de producción nacionales (Sánchez y Giraldo, 2008). De esta forma, aunque las mipymes colombianas se diferencian de las medianas y pequeñas empresas en el mundo por su tamaño $y$ 
otras variables cuantitativas, tales como el número de empleados, el nivel de ventas y el total de activos; presentan elementos similares independiente del lugar geográfico en que se encuentren. En este sentido, se consideran «el motor de la economía, por su aporte al crecimiento del PIB, la generación de empleo, el aumento de la productividad y la capacidad de cambio e innovación» (Sánchez y Giraldo, 2008, p. 428).

Ahora bien, para el caso de las mipymes del sector servicios, el Censo Económico de 2005 del DANE (Ministerio de Comercio, Industria y Turismo y Viceministerio de Desarrollo Empresarial, 2008) señala que cuentan con un $33,7 \%$ de la participación productiva en la actividad económica nacional de Colombia. Esto refleja que este sector ha ganado dinamismo en su crecimiento y cada vez representa mayores oportunidades de negocios para los empresarios. Actualmente, en Colombia, el sector servicios genera diversos impactos en sus operaciones, principalmente en razón a sus resultados en términos económicos, culturales, sociales y ambientales. En el tópico social la generación de empleo se ha registrado como una tendencia que a futuro representará la ocupación de millones de ciudadanos.

Según el Ministerio de Comercio, Industria y Turismo, las microempresas en Colombia se dedican en un $53,4 \%$ a actividades del comercio al por menor relacionadas con alimentos, prendas de vestir y accesorios, además de servicios vinculados con comidas preparadas, restaurantes y cafeterías (2008).

Las pequeñas y medianas empresas del sector servicios en Colombia realizan, especialmente, actividades de práctica médica, educación básica y secundaria y actividades relacionadas con instituciones prestadoras de salud. Según el Centro de Estudios Económicos de la Asociación Nacional de Instituciones Financieras de Colombia, en el 2018 los empresarios del sector de servicios mejoraron su situación económica en términos generales.
No obstante, los empresarios de las mipymes señalan que existe una serie de problemas que los aquejan. Los principales son la competencia, los altos impuestos, la falta de demanda y la rotación de cartera. De acuerdo con el Centro de Estudios Económicos, «en las pequeñas empresas el principal problema fue la competencia, mientras que en las medianas fueron los altos impuestos» $(2018$, p. 17). Así mismo, Andriani y Col (como se citan en Vera y Mora, 2011) identifican y agrupan las deficiencias que presentan las mipymes en cuatro aspectos: «Sistema de gestión sin direccionamiento claro, desorden e improvisación en los procesos, desmotivación y deshumanización de sus recursos humanos y falta de un sistema de información y diagnóstico adaptado a su situación» (p. 220).

Por su parte, Sánchez y Giraldo (2008) señalan que las pymes han atendido poco la generación e implementación de herramientas de gestión para la formalización y el fortalecimiento de los procedimientos propios de la organización, lo cual hace que se vean limitadas por la informalidad en su estructura interna y en el desarrollo de sus operaciones. Esto, unido a la falta de infraestructura tecnológica y de producción, las hace menos competitivas.

\subsection{Principales hallazgos sobre la gestión del talento en micro, pequeñas y medianas empresas colombianas}

Para las grandes empresas, las políticas y las prácticas en temas vinculados con la gestión del talento humano han sido motivo de innumerables estudios. Sin embargo, poco se ha investigado acerca de este tipo de prácticas en las pequeñas empresas (Rodríguez, 2012). Entre los principales hallazgos de estos estudios se destaca cómo, en la medida en que aumenta el tamaño de la organización, existe mayor conciencia sobre la importancia del capital humano y su contribución a la estrategia del negocio. Calderón añade que aun cuando existe más valor agregado en las empresas grandes que en las pequeñas, estadísticamente no hay una diferencia significativa entre ellas. Del mismo modo, señala: 
La dimensión donde más aporte hacen las empresas grandes y medianas es la estratégica; las pequeñas tienen su aporte más crítico en infraestructura organizacional. A diferencia del tamaño, por sector económico sí existe diferencia significativa en el valor agregado: el sector servicios tiene un promedio de valor agregado inferior a los sectores industria $y$ comercio. (2008, p. 36)

En esta misma línea, Rodríguez (2012) plantea que, para los pequeños empresarios, las prácticas de gestión humana carecen de visión estratégica $y$ tienen un sentido puramente operativo, lo cual se presenta también en la mediana empresa. Las pequeñas empresas no se sobreponen a lo operativo del día a día y eso conduce a que tengan escasa conciencia sobre los beneficios de la coordinación de la gestión estratégica de las personas con la estrategia organizacional general.

Para el caso de las pequeñas empresas se evidencia que, en la mayoría de los casos, no cuentan con áreas especializadas de gestión del talento humano, pero sí cuentan con áreas financieras y comerciales. Esto se contrapone a las consideraciones de los directivos de este tipo de organizaciones, quienes consideran que las personas son claves para el éxito de la organización. De esta manera, existe una incoherencia entre sus planteamientos y sus prácticas en la organización (Rodríguez, 2012).

Calderón (2008) añade que las empresas medianas y algunas grandes son conscientes de los nuevos retos organizacionales en materia de gestión del talento y han iniciado procesos de cambio, pero aún encuentran limitaciones propias de gestión humana pues son todavía muy operativas y no han logrado externalizar procesos tales como nómina o salud ocupacional con el fin de hacer más eficiente la operación. Esto limita sus capacidades de agregar valor $y$ las orienta a tener actitudes poco positivas hacia las personas, ya que las consideran un gasto y no un capital por desconocimiento de la importancia de la gestión humana en la organización.

Calderón (2008) también señala que existe otro grupo de organizaciones conformadas, en su mayoría, por la pequeña empresa y algunas medianas, en el que la gestión humana carece de posicionamiento y se centra en aspectos operativos y administrativos. Esto genera que cuando se da algún aporte de valor este no es significativo frente a exigencias de competitividad. Al respecto, Rodríguez (2012) explica: «Las prácticas de recursos humanos en pequeñas empresas son informales y más flexibles comparadas con las grandes, usualmente se usan para monitoreo y control en lugar de propósitos de desarrollo» (p. 209). Agrega que las nuevas y pequeñas empresas pueden tener mayor dificultad para reclutar y retener trabajadores debido a la falta de políticas y sistemas de gestión humana. Con relación a las prácticas de aprovisionamiento de personas, Calderón et al. (2010) añaden que la falta de posicionamiento estratégico en la selección de personas en las mipymes se debe a que en este tipo de organizaciones predominan criterios basados en las referencias de los empleados, a diferencia de las grandes empresas en las que prevalecen razones fundadas en criterios socioculturales y técnicos.

En relación con el reclutamiento y la selección, Rodríguez (2012) suma que las medianas y pequeñas empresas, así como las microempresas, utilizan fuentes informales, como, por ejemplo, familiares y amigos, $y$ «predominan procesos intuitivos centrados en entrevistas y certificaciones, pero poco se acude a pruebas técnicas. Entonces, en este tipo de organizaciones los criterios socioculturales prevalecen sobre criterios técnicos a la hora de vincular a una persona» (p. 214).

Ahora, con relación a la formación y la capacitación, otro de los procesos centrales de la gestión del talento humano, en específico para las empresas colombianas, Calderón et al. (2011) señalan que «las limitaciones de las empresas colombianas en el tema de capacitación, incluso en la forma en que la conciben actualmente, son evidentes» (p. 42). Sobre lo anterior, el estudio del Corpes (1995) encontró que el $41 \%$ de las empresas colombianas no planificaba la formación de su capital humano, y las que lo hacían tenían un horizonte temporal inferior a un año. Las investigaciones señalan que 
la pequeña y mediana empresa carece de programas de capacitación estructurados al ser limitada la inversión que desembolsan para la formación de sus empleados. Del mismo modo, la evidencia advierte que el entrenamiento en las pequeñas empresas es informal y se efectúa en el puesto de trabajo, es decir, mientras las personas realizan sus funciones. Entonces, estas organizaciones, «raramente llevan un análisis de necesidades de formación. La capacitación se percibe como un lujo y un costo en tiempo productivo». (Rodríguez, 2012, p. 215). Los hallazgos indican también cómo en Colombia se invierte poco en formación porque las empresas esperan que el trabajador, al ingresar a la organización, aporte los conocimientos requeridos.

Por otra parte, respecto al sistema de compensaciones, Calderón et al. (2010) plantean que existe un grado de desarrollo incipiente en las pequeñas y medianas empresas en relación con este aspecto, y señalan que en Colombia estas prácticas se limitan al cumplimiento de la ley y carecen de una visión estratégica que las oriente hacia el rendimiento y la consecución de objetivos y metas, así como a la generación de compromiso y motivación de los empleados. En otros estudios sobre prácticas retributivas estos autores lograron evidenciar que la remuneración en Colombia se expresa en beneficios $y$ no en dinero en efectivo, y que es el seguro de vida el beneficio que más se utiliza en la alta gerencia, seguido de los planes médicos y los planes de vehículos (Calderón et al., 2011).

Investigaciones sobre las prácticas de compensación en Colombia también indican que «son escasas las empresas que tienen establecidos mecanismos de reconocimiento tales como premios, diplomas, mención pública, fiestas, paseos y celebraciones» (Rodríguez, 2012, p. 217). Mientras que, en cuanto a la evaluación del desempeño, las investigaciones indican que las empresas pequeñas realizan la evaluación de manera informal, y la orientan, principalmente, a la búsqueda de problemas y dificultades y no al encuentro de fortalezas o potencialidades. Del mismo modo, los hallazgos en las mipymes sobre estas prácticas plantean que la evaluación del desempeño no se utiliza para gestionar competencias ni con el fin de diseñar planes de entrenamiento y capacitación, sino que se usa con el propósito de vincularla a los sistemas de retribución (Calderón et al., 2010). Sobre estos procesos se ha hallado que en las pequeñas empresas la evaluación del desempeño se asocia a la detección de dificultades en el trabajo, $y$, en casos excepcionales, a la búsqueda de fortalezas. Poco se utiliza para retroalimentar al trabajador, programar capacitaciones, mejorar procesos o realizar promociones. Además, al igual que en las otras prácticas de gestión del talento humano, existe un predominio de criterios subjetivos e intuitivos para realizar la evaluación del desempeño (Calderón y Álvarez, 2006, como se citan en Rodríguez, 2012, p. 218; DeNisi, 2017).

En cuanto a planes de carrera y promoción, los hallazgos indican que, en la mayoría de las pequeñas y medianas empresas colombianas, no existen planes de carrera formalmente establecidos, pues la estructura organizacional de pequeño tamaño no permite establecer ascensos (Rodríguez, 2012).

A pesar de las dificultades que evidencian las mipymes en el desarrollo de prácticas de gestión del talento humano para agregar valor a la empresa, existen estudios sobre mipymes que señalan rasgos que constituyen nuevas ventajas competitivas para ellas en comparación con las grandes empresas. La más relevante es la flexibilidad de las mipymes, lo que posibilita una mayor aproximación a los clientes y una respuesta más rápida y exacta a sus necesidades, además de una mayor orientación al mercado (Goldman y Nagel 1993; Stalk, Evans y Shulman, 1992; como se citan en Camisón, 1996).

Adicionalmente, estos estudios señalan que existen ciertos factores relacionados con los recursos humanos que pueden ser una fuente de ventaja competitiva para las mipymes:

\footnotetext{
La difusión de las nuevas tecnologías y el rápido cambio de los conocimientos colocan la formación y el reciclaje como acción fundamental para acompasar la innovación tecnológica y la competitividad
} 
humana.Y también, junto a la necesidad de esforzarse para evitar la obsolescencia de las habilidades y competencias del personal, otro factor de cambio transcendental son nuevos principios de gestión de los recursos humanos que hacen insistencia en la participación, compromiso y la implicación del trabajador en la institución. (Camisón, 1996, p. 83)

Finalmente, la evidencia bibliográfica disponible en estos estudios ha constatado que el fracaso en muchas pequeñas empresas, a pesar de contar con buenos productos, se debe a su falta de activos complementarios, especialmente intangibles. En particular, las investigaciones han establecido que la debilidad más preocupante de las mipymes se basa en la capacidad de su personal y la dirección, y es la capacidad directiva la más crítica en lo relacionado con la inexperiencia y la incompetencia administrativa para conducir el negocio (Pumplin y García Echeverría 1993, como se citan en Camisón, 1996).

\subsection{Aproximaciones a un modelo de diagnós- tico organizacional sobre gestión del talento humano}

A la hora de explicar la generación y mantención de las ventajas competitivas en las empresas, existen dos grandes teorías. Una es la cadena de valor elaborada por el ingeniero y profesor Michael Porter, académico de la Escuela de Negocios de Harvard. La otra es la teoría de recursos y capacidades de Wernerfelt, de 1984, perfeccionada por Barney en 1991 (Reynoso, Flores y Cardoza, 2017).

El enfoque de la cadena de valor plantea que las empresas deben orientarse a una determinada ventaja competitiva para mantenerse en el mercado. Al respecto, Porter señala que «una empresa obtiene la ventaja competitiva desempeñando estas actividades - de la cadena de valor- estratégicamente importantes más baratas o mejores que sus competidores» (De la Fuente y Muñoz, 2003, p. 4).

Mientras que la teoría de recursos y capacidades explica cómo el éxito de las empresas recae en las características de los recursos y las capacidades que controlan, así como en la posibilidad que tienen para generar rentas. Esta teoría, del mismo modo, señala que una eficiente combinación de los recursos y las capacidades de las empresas permite la obtención y el mantenimiento de su ventaja competitiva (Reynoso, 2017).

En términos genéricos, la cadena de valor establece que las actividades primarias - logística de entrada, producción, logística de salida, marketing y ventas, servicio de posventa- y de apoyo que sustentan a las actividades primarias — abastecimiento, investigación y desarrollo, administración de recursos humanos, infraestructura de la empresa-, son una fuente clave de ventaja competitiva en la medida que se integran de forma entrelazada para generar valor en el sistema total —empresa-(Hill y Jones, 1996).

Ahora bien, según De la Fuente y Muñoz (2003) $\ll$ la diferencia entre recursos y actividades radica en que los recursos se asocian al concepto de stock, mientras que las actividades se asocian más bien al concepto de flujos. La tesis principal de este pensamiento es que las empresas tienen desempeños distintos porque no tienen el mismo stock de recursos. De esta forma, las ventajas competitivas surgen porque existen recursos valiosos que están en posesión solo de uno o unos pocos participantes del mercado (Hill y Jones, 1996; Hitt et al., 1999).

De la Fuente y Muñoz clasifican los recursos en tres grandes categorías:

- Activos tangibles. Aquellos activos que pueden ubicarse con mayor facilidad, ya que, por lo general, pueden encontrarse reflejados en el balance general de una empresa. Este es el caso de las maquinarias, los equipos, las plantas productivas, las oficinas de venta, fondos financieros, etc.

- Activos intangibles. Son recursos que se caracterizan por no ser consumidos por su uso, como es el caso de los activos tangibles. A diferencia de estos últimos, pueden acrecentar su valor con el transcurso del tiempo. Entre estos tenemos la 
imagen de marca, la cultura organizacional, las patentes y las experiencias acumuladas.

- Capacidades organizacionales. Son complejas combinaciones de activos, personas y procesos que las organizaciones utilizan para transformar los insumos en productos y servicios (De la Fuente y Muñoz, 2003, p. 7).

Respecto a los activos intangibles y las capacidades organizacionales, Calderón plantea:

El área de gestión humana es considerada como un recurso organizacional que clasifica dentro de los activos intangibles, importante en el término del logro de capacidades distintivas de la empresa. Por cuanto, no basta implementar prácticas de alto rendimiento si no existe detrás de ellas una dirección de gestión humana bien estructurada que las respalde, las integre y las sustente. (2006, p. 25)

Con base en el enfoque de recursos y capacidades, Calderón (2008) propone un modelo de gestión humana para establecer el valor agregado que la gestión humana entrega a las áreas de las organizaciones. Este modelo lo puede implantar cualquier organización y lo adaptan «empresas de diversos sectores, tamaños y filosofía empresarial» (p. 78).

La fórmula de Calderón considera cinco dimensiones: proyección organizacional —orientación estratégica一, gestión del cambio, infraestructura organizacional —eficiencia y eficacia-, liderazgo de las personas y responsabilidad social.

La proyección organizacional se refiere a la capacidad del área de gestión humana para generar políticas de valor estratégico que respondan a problemas críticos de la organización y la generación de objetivos y metas para resolverlos. También considera el análisis del entorno y el desarrollo de mejores prácticas para la optimización de procesos, lo que se concreta en proyectos internos de mejoramiento con el respectivo soporte desde la gestión humana (Calderón, 2008).

La gestión del cambio, por su parte, trata sobre la identificación de necesidades de cambio, la importancia de impactar la cultura organizacional y la movilización para el cambio. En estas acciones el rol de los directivos de las áreas de gestión humana es decisivo para lograr que la alta dirección se comprometa con el cambio.

La infraestructura organizacional tiene que ver con el valor que se agrega a través de procesos funcionales y administrativos - selección, formación, evaluación, compensación-, diseñados y ejecutados con visión estratégica, en el propósito de fomentar la eficiencia y eficacia organizacional. Esto considera la medición de resultados y la búsqueda de métodos alternativos para la organización, así como la realización del trabajo. Señala Calderón:

$$
\begin{aligned}
& \text { Una adecuada infraestructura organizacional se } \\
& \text { asocia a buenos resultados en productividad. } \\
& \text { Para lograrla, se requiere una organización del } \\
& \text { trabajo que conlleve enriquecimiento de la tarea, } \\
& \text { sistemas apropiados de supervisión, óptima } \\
& \text { comunicación, mediciones del rendimiento, } \\
& \text { flexibilidad y métodos de trabajo que permitan } \\
& \text { eliminar desperdicios, cuellos de botella y trabajo } \\
& \text { innecesario. (2008, p. 63) }
\end{aligned}
$$

El liderazgo de las personas lo entiende como la capacidad de lograr una contribución discrecional del trabajador. Calderón manifiesta que esta es condición de la dirección de las empresas para que los resultados de quien la gerencia estén vinculados con los resultados del equipo con el cual trabaja y la capacidad para crear las condiciones que le permitan movilizar a las personas y lograr su contribución.

Además, la última dimensión del modelo se refiere a la responsabilidad social, entendida como la preocupación no solo de los problemas de crecimiento económico y la eficiencia en el uso de sus recursos, sino también los de su entorno, al intervenir en problemas de salud, educación, empleo, contaminación o cultura y equidad, entre otros.

Con base en la experiencia y la revisión de otros modelos analíticos sobre gestión estratégica de recursos humanos o gestión humana, se considera pertinente complementar el modelo propuesto por Calderón sobre el valor agregado de las áreas de gestión humana en las organizaciones y adicionar 
dos dimensiones necesarias para comprender la contribución de las áreas de gestión humana a los resultados organizacionales.

La primera dimensión corresponde a los recursos tecnológicos de los que deben disponer las áreas de gestión humana a fin de cumplir su misión y agregar valor a la organización. Se consideran para este ítem el equipamiento en tecnologías de información y comunicación, las herramientas de software y las competencias del personal del área sobre nuevas tecnologías (Dirección Nacional del Servicio Civil, 2006). Las áreas de gestión humana deberían estar alineadas de forma directa con la estrategia organizacional, desde una perspectiva sistémica para que todos los subsistemas de la organización, y no solo la gestión de personas, estén dispuestos a funcionar y conseguir lo que la organización necesita. En este sentido, «las tecnologías como medios o soportes institucionales también deben enfocarse hacia este objetivo y constituyen un valioso recurso para que la organización realice más eficientemente su labor, pero deben ser utilizados integradamente y coherentemente» (Dirección Nacional del Servicio Civil, 2007, p. 5).

Más allá del software y el hardware debe existir una madurez de soporte tecnológico que permita responder integralmente a las necesidades del negocio, principalmente porque la nueva era digital exige la inversión en el desarrollo de capacidades digitales que deben estar muy bien alineadas a la estrategia de la empresa:

El desarrollo de estas capacidades es el pilar para mantener a la empresa en la carrera por satisfacer a los clientes en un entorno que está cambiando la manera cómo los clientes investigan sobre los productos o servicios, compran a través de medios digitales o se interrelacionan con el mundo. El desarrollo de estas capacidades debe ocurrir de manera integrada en todas las dimensiones de la organización: estrategia, personas y cultura, estructura y sistemas de gestión, procesos de negocio y, por supuesto, en la tecnología. (Ochoa, 2016, p. 574)
En un estudio realizado entre el 2014 y el 2015 por McKinsey a 150 empresas a nivel global dirigido a comprender los retos de la digitalización en las organizaciones (Catlin, Scanlan y Willmott, 2015, como se citan en Ochoa, 2016), se estableció que a fin de alcanzar el éxito digital era crítico construir bases para otras capacidades claves asociadas a procesos y actividades. De esta forma, se concluyó que la habilidad de comprometer a los clientes de manera digital y mejorar el desempeño de costos de la empresa se basa en cuatro áreas: a) toma de decisiones guiada por datos y evidencias; $b$ ) conectividad, en cuanto uso de la tecnología para desplegar una relación y unas conexiones más profundas entre las marcas y los clientes; c) automatización de procesos claves del negocio; y d) tecnologías de información diseñadas para entregar resultados rápidos a los clientes y optimizar las operaciones tradicionales y del back-office de la organización.

La segunda dimensión adicional que se propone tiene que ver con la posición y la influencia del área de gestión humana en el interior de la empresa, referida al nivel de incidencia relativa de esta área a nivel organizacional, es decir, en la posición jerárquica que ocupa en la organización y en el acceso a la toma de decisiones que tiene (Dirección Nacional del Servicio Civil, 2007). Habrá mayor contribución del área a los objetivos organizacionales si cuenta con un posicionamiento tal que le permita acceder a la toma de decisiones estatégicas.

Sobre las implicancias de la dimensión relacionada con la posición e influencia del área de gestión humana, un estudio desarrollado en Chile en el 2005 con 160 servicios públicos del nivel central del Estado de Chile, a fin de diagnosticar el estado de las unidades de recursos humanos (URH) de las instituciones públicas, estableció lo siguiente:

El desempeño exitoso de las URH, incide fuertemente en el posicionamiento de la jefatura de estas unidades y el lugar que la misma ocupa en la jerarquía institucional. Pero, además, se relaciona con la convicción del jefe superior del servicio respecto del rol estratégico que debe jugar la unidad en el desempeño del servicio. La visión política de 
la jefatura se vuelve fundamental para colocar los temas de gestión de personas entre los objetivos centrales de la institución. La jefatura de estas unidades, en suma, deben compatibilizar el rol técnico con el rol político-estratégico. (Dirección Nacional del Servicio Civil, 2006, p. 29)

Del mismo modo, se constató que los altos directivos de las organizaciones que demostraban un compromiso activo y eficiente en la promoción y el desarrollo de las personas incorporaban objetivos estratégicos y metas específicas relacionadas con la gestión humana. Esto, al integrar a los directivos de las áreas de recursos humanos en los comités en los que se discutían y tomaban decisiones, y al mantener una línea directa y frecuente con el área, lo que les permitía asumir roles estratégicos de apoyo para un mayor desarrollo global de la gestión de la institución (Dirección Nacional del Servicio Civil, 2006).

Otro estudio de la Dirección Nacional del Servicio Civil de Chile, desarrollado en el 2017 en 232 servicios públicos, dio cuenta de una medición periódica sobre el avance en la gestión y el desarrollo de personas en el interior de las instituciones públicas. En esta investigación se estableció que, cuando la jefatura del área de recursos humanos no participaba en instancias de reuniones del Comité Directivo de la institución, el área de gestión de personas alcanzaba un nivel de desarrollo inferior que cuando participaba ocasional o permanentemente:

$$
\begin{aligned}
& \text { Estas diferencias plantean que la visibilidad de } \\
& \text { las temáticas del Área de Gestión de Personas, en } \\
& \text { relación con la toma de decisiones para el logro } \\
& \text { de los objetivos estratégicos de cada institución, } \\
& \text { potencia el nivel de desarrollo del área y su aporte } \\
& \text { a las necesidades institucionales. (Dirección } \\
& \text { Nacional del Servicio Civil, 2017, p. 20) }
\end{aligned}
$$

En consecuencia, los estudios mencionados demuestran que la contribución de las áreas de gestión humana a los resultados del negocio depende de múltiples factores que deben observarse y analizarse desde una perspectiva sistémica como un todo y de forma complementaria.

\section{Conclusiones}

$\mathrm{E}$ la actualidad, las áreas de gestión del talento humano constituyen un recurso organizacional fundamental entre los activos intangibles de las empresas que influyen en la composición de sus capacidades distintivas y competitivas a fin de enfrentar escenarios complejos.

Las áreas de gestión del talento generan valor en las organizaciones en cinco dimensiones: proyección organizacional —orientación estratégica一, gestión del cambio, infraestructura organizacional eficiencia y eficacia—, liderazgo de las personas y responsabilidad social. De modo complementario, los recursos tecnológicos y la posición e influencia de estas áreas en la estructura organizacional pueden constituir un valioso recurso para el funcionamiento de las empresas y el cumplimiento de sus objetivos estratégicos.
En las mipymes colombianas las áreas de gestión del talento humano pueden erigirse como una herramienta movilizadora de recursos internos y en fuente de ventaja competitiva, en la medida en que se integren, de forma entrelazada, en el propósito de generar valor en la organización como un sistema integral.

El principio básico de soporte para la competitividad de las mipymes colombianas es el desarrollo de estilos de gestión directiva orientados al análisis y la comprensión de escenarios complejos y heterogéneos, así como la implementación de estrategias flexibles y oportunas.

A fin de enfrentar estos desafíos la alta gerencia debe asesorarse por áreas de gestión del talento humano que la respalden, integren y brinden soporte, $y$ sean capaces de implementar políticas y prácticas de gestión y desarrollo de personas alineadas a la estrategia de la organización. 


\section{Referencias}

Calderón, G. (2006). La gestión humana y sus aportes a las organizaciones colombianas. Revista Cuadernos de Administración, 19(31), 9-55. Recuperado de http://www. redalyc.org/pdf/205/20503102.pdf

Calderón, G. (2008). Aproximación a un modelo de gestión humana que agregue valor a la empresa colombiana. Colombia: Universidad Nacional de Colombia sede Manizales, Manuales Facultad de Administración, Grupo de Investigación Cultura Organizacional y Gestión Humana.

Calderón, G.; Naranjo, J.; Álvarez, C. (2010). Gestión humana en la empresa colombiana: sus características, retos y aportes. Una aproximación a un sistema integral. Revista Cuadernos de Administración, 23(41), 13-36. Recuperado de http://www.redalyc.org/pdf/205/20516983002.pdf

Calderón, G.; Naranjo, J.; Álvarez, C. (2011). Gestión empresarial en Colombia: un aporte desde la administración. Colombia: Universidad Nacional de Colombia, Vicerrectoría Académica Editorial.

Camisón, C. (1996). Competitividad y teoría de la estrategia un análisis aplicado a la cohabitación pyme-gran empresa. Revista Asturiana de Economía, (6), 63-101.

Centro de Estudios Económicos. (2018). La gran encuesta pyme. Lectura nacional año 2018. Colombia: ANIF. Recuperado de https://bit.ly/2QEnEmQ

Cuesta, A. (2017). Gestión del talento humano y del conocimiento. Colombia: Ecoe Ediciones.

De la Fuente, M.; Muñoz, C. (2003). Ventaja competitiva: ¿actividades o recursos? Revista Panorama Socioeconómico, (26), 1-14. Recuperado de http://www.redalyc.org/ articulo.oa? $\mathrm{id}=39902603$

DeNisi, A. (2017). Performance appraisal and performance management: 100 years of progress? Journal of Applied Psychology American Psychological Association, 102(3), 421-433. DOI: https://doi.org/10.1037/apl0000085

Dirección Nacional del Servicio Civil. (2006). Informe ejecutivo diagnóstico de las unidades de recursos humanos de los servicios públicos, año 2006. Gobierno de Chile, Ministerio de Hacienda Servicio Civil. Recuperado de https://bit. ly/3dqUYaL

Dirección Nacional del Servicio Civil. (2007). Informe ejecutivo diagnóstico de las tecnologías de información para la gestión de recursos humanos en los servicios públicos, año 2007. Gobierno de Chile, Dirección Nacional del Servicio Civil. Recuperado de https://bit.ly/2Jb4K31

Dirección Nacional del Servicio Civil. (2017). Informe de resultados barómetro gestión de personas de la administración central del Estado, actualizado con resultados de Instructivo Presidencial sobre Buenas Prácticas Laborales en Desarrollo de Personas en el Estado. Informe Global, año 2017. Gobierno de Chile. Recuperado de https://bit.ly/2UxlpD4
Hill, C.; Jones, G. (1996). Administración estratégica: un enfoque integrado. Colombia: Editorial McGraw-Hill.

Hitt, M.; Ireland, D; Hoskisson, R. (1999). Administración estratégica: competitividad y conceptos de globalización. México: International Thomson Editores.

Koontz, H.; Weihrich, H.; Cannice, M. (2004). Administración: una perspectiva global. México: Editorial McGraw-Hill.

Ley 905 de 2004. (2 de agosto de 2004). Sobre promoción del desarrollo de la micro, pequeña y mediana empresa colombiana y se dictan otras disposiciones. Diario Oficial núm. 45628. Congreso de Colombia.

Ministerio de Comercio, Industria y Turismo y Viceministerio de Desarrollo Empresarial, Dirección de Mipymes. (2008). Reporte de mipymes N. 3 , año 2008. Colombia. Recuperado de http://www.mincit.gov.co/servicio-al-ciudadano/ preguntas-frecuentes/mipymes

Ochoa, O. (2016). Modelos de madurez digital: ¿en qué consisten y qué podemos aprender de ellos? Boletín de Estudios Económicos, 71(219), 573-590. Recuperado de https://issuu.com/deustobusinessalumni/docs/ boletin 219

Pfeffer, J. (2000). Nuevos rumbos en la teoría de la organización: problemas y posibilidades. México: Oxford University Press.

Reynoso, C.; Flores, K.; Cardoza, L. (2017). La teoría de recursos y capacidades: un análisis bibliométrico. Revista Electrónica Nova Scientia, 9(2), 411-440. DOI: https://doi. org/10.21640/ns.v9i19.739

Rodríguez, D. (2012). Prácticas de gestión humana en pequeñas empresas. Revista Apuntes del Cenes, 54(31), 193-226. DOI: https://doi.org/10.19053/22565779.21

Sánchez, A.; Giraldo, N. (2008). Las necesidades de las pymes - pequeñas y medianas empresas - y el sistema de información contable y financiero como una estrategia para atenderlas. Revista Cuadernos de Contabilidad, 9(25) 421-464. Recuperado de https://revistas.javeriana.edu.co/ index.php/cuacont/article/view/3232

Tejada, A. (2003). Los modelos actuales de gestión en las organizaciones. Gestión del talento, gestión del conocimiento y gestión por competencias. Revista Psicología desde el Caribe, 12, 115-133. Recuperado de http://www. redalyc.org/articulo.oa? $\mathrm{id}=21301208$

Ulrich, D. (1998). Recursos humanos champions. Argentina: Ediciones Granica.

Vera, M.; Mora, E. (2011). Líneas de investigación en micro, pequeñas y medianas empresas. Revisión documental y desarrollo en Colombia. Revista Tendencias de la Facultad de Ciencias Económicas y Administrativas, 12(1), 213-226. 